\title{
Aktuelles in der Frühtherapie der Säuglingshämangiome
}

\section{The Current of the Early Treatment of Hemangiomas in Infancy}

\section{Autor}

Institut

\section{Dj. Djawari}

Hautklinik der SLK Kliniken Heilbronn (Direktor: Prof. Dr. med. Dj. Djawari), Akademisches Lehrkrankenhaus der Universität Heidelberg

\section{Bibliografie}

DOI $10.1055 / \mathrm{s}-2006-944561$

Akt Dermatol 2006; 32;

418-420 @ Georg Thieme

Verlag KG Stuttgart · New York ISSN 0340-2541

Korrespondenzadresse Prof. Dr. med. Dj. Djawari Direktor der Hautklinik, SLK Kliniken Heilbronn Klinikum am Gesundbrunnen 74078 Heilbronn djalil.djawari@slk-kliniken.de

\section{Zusammenfassung}

$\nabla$

Seit mehr als 15 Jahren werden die Säuglingshämangiome einer Frühtherapie unterzogen. Die abwartende Haltung, die früher als Lehrmeinung galt, ist inzwischen obsolet. Die Säuglings-

Säuglingshämangiome sind Gefäßneubildungen, die erst nach der Geburt und nach einem kurzen Prodromalstadium im ersten Lebensjahr, überwiegend jedoch in der 6.-8. Lebenswoche stark proliferieren. Die Wachstumsphase dieser gutartigen Neubildungen dauert bis zum Ende des ersten Lebensjahres. Danach kommt es zu einer langsamen Rückbildung, die Jahre, nicht selten sogar bis zur Pubertät andauern kann. Nicht hierzu gehören die Gefäßfehlbildungen (vaskuläre Malformationen, z.B. Naevi flammei), die seit der Geburt bestehen, mitwachsen und sich nicht zurückbilden.

Die meisten Säuglingshämangiome sind im Kopfund Halsbereich bzw. anogenital lokalisiert. 20\% dieser Anomalien zeigen keine und weitere 30\% nur eine unvollständige Regression. Hämangiome im Lippen- und Nasenbereich bilden sich nur ausnahmsweise spontan zurück. Morphologisch können nach der neuen Nomenklatur oberflächliche, tiefe und gemischte Hämangiome unterschieden werden.

Durch die früher übliche abwartende Haltung als Lehrmeinung bei der Therapie der Säuglingshämangiome ging viel Zeit in der frühen Prodromal-bzw. Wachstumsphase dieser Hautveränderungen verloren. Gerade in dieser Phase der Neubildung ist jede Therapie sehr effektiv.

Um den betroffenen Patienten und ihren Familien jahrelange kosmetische wie auch psychische Belastungen zu ersparen und vor allem den Komplikationen vorzubeugen, wird nun seit über 15 Jahren die Frühtherapie der Säuglingshämangio- hämangiome werden sehr früh und in der Entstehungs- bzw. Wachstumsphase effektiv behandelt. Den kleinen Patienten und deren Familien bleiben dadurch psychosoziale und vor allem medizinische Nachteile erspart.

me gleich in der Entstehungs-, Prodromal- und spätestens in der Wachstumsphase praktiziert. In Anbetracht der Weiterentwicklung gewebsschonender Therapieverfahren bei der Frühbehandlung von Säuglingshämangiomen gehört die praktizierte nihilistische Lehrmeinung einer abwartenden Haltung nun der Vergangenheit an. Die unberechenbar wachsenden Säuglingshämangiome werden durch die Frühtherapie erfasst und in der Anfangsphase des Wachstums effektiv behandelt. Wir sind froh, dass die Frühbehandlung der Säuglingshämangiome sich durchgesetzt hat und damit vielen Kindern bzw. ihren Familien psychosoziale Belastungen oder auch die medizinischen Gefahren (Schädigung benachbarter Organe) erspart blieben.

Es ist nun sekundär, welche Therapieform, ob Laserbehandlung oder Kryochirurgie gewählt wird. Wichtig ist, dass die Säuglingshämangiome frühzeitig vorgestellt und diese in der Entstehungsbzw. in der Wachstumsphase behandelt werden. Durch die Lasertherapie wird das Wachstum der Hämangiome blockiert und die Regression erfolgt schneller.

Mit der Kryochirurgie wird nicht nur das Wachstum blockiert, sondern auch das ganze Hämangiom zerstört und beseitigt. Nach der Kryochirurgie kommt es zu einer Mumifizierung und danach zum Abfallen des krustig-nekrotischen Hämangioms. Nach zwei Wochen sieht man nur

\footnotetext{
${ }^{*}$ Herrn Prof. Dr. med. O. P. Hornstein zum 80. Geburtstag gewidmet, teilweise beim wissenschaftlichen Symposium am 28.1.2006 zu diesem Anlass in Erlangen präsentiert.
} 

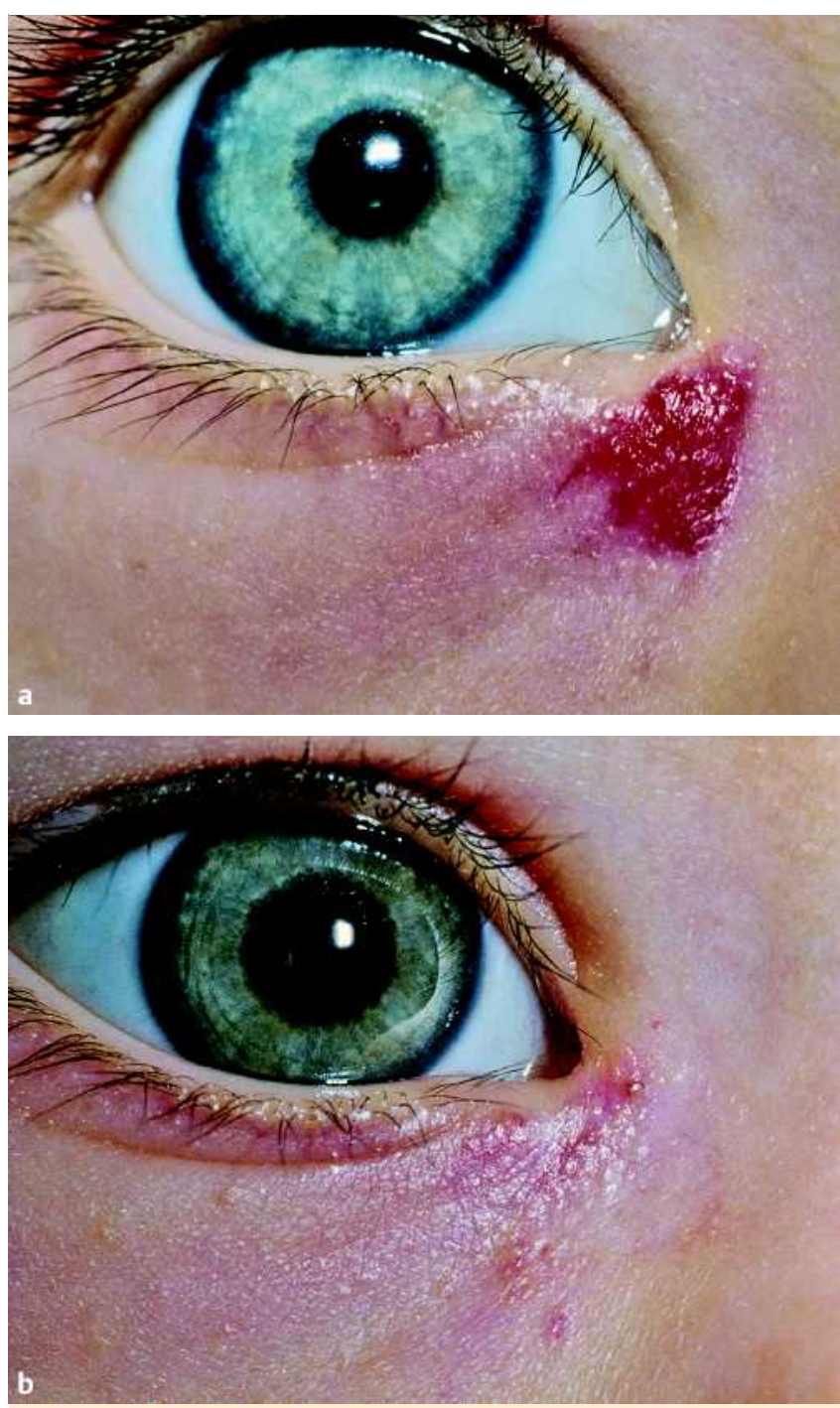

Abb. 1 Beispiel eines Säuglingshämangioms. a vor, und b nach der kontaktkryochirurgischen Therapie.

noch eine geringe entzündliche Rötung und nach weiteren zwei Wochen eine vollständige Abheilung.

Aus diesem Grund ziehen wir weiterhin die Kryochirurgie der Säuglingshämangiome anderen therapeutischen Verfahren vor. Die Ergebnisse der Kryochirurgie sprechen nun nach mehr als $1 \frac{1}{2}$ Jahrzehnten in Konkurrenz zu den Lasermethoden für sich. ( $\triangle$ Abb. 1 u. 2). Natürlich gibt es auch hier Grenzen, die beachtet werden müssen. Tiefliegende Hämangiome können kryochirurgisch nicht erreicht und nicht behandelt werden. Bei den großflächigen Säuglingshämangiomen empfehlen wir die Lasertherapie. Unsere weiterhin gültige Regel bei der Frühtherapie der Säuglingshämangiome ist in der $\bullet$ Abb. 3 dargestellt.

Für ein 100\%ig effektives Resultat der Kryochirurgie müssen drei grundlegende Faktoren berücksichtigt werden:

1. die Applikationstemperatur,

2. die Applikationsdauer,

3. der Applikationsdruck.

Ideale Ergebnisse erzielen wir bei einer Temperatur von minus $196^{\circ} \mathrm{C}$ (flüssiger Stickstoff), bei einer Therapiedauer von $10 \mathrm{Se}-$ kunden und bei einem moderaten, den Lokalisationsverhältnissen angepassten Druck (Metallstempel).
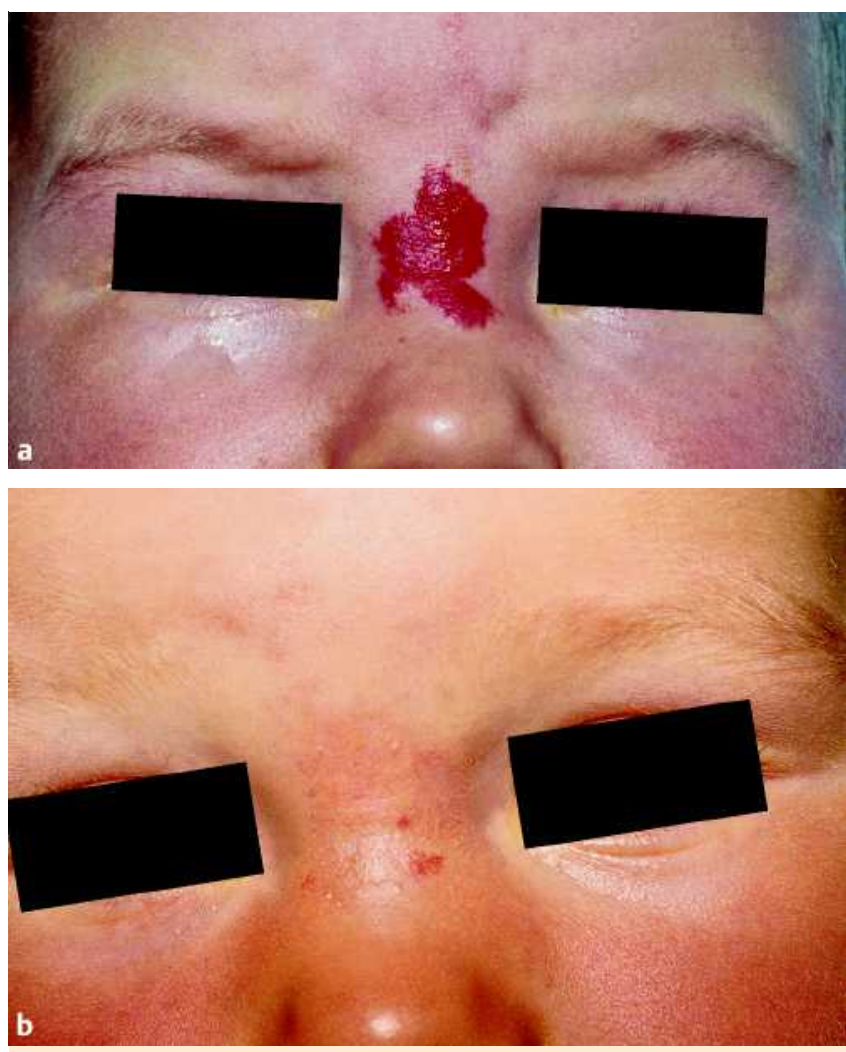

Abb. 2 Beispiel eines Säuglingshämangioms. a vor, und $\mathbf{b}$ nach der kontaktkryochirurgischen Therapie.

Alle nach dieser Methode rechtzeitig bzw. früh behandelten Säuglingshämangiome zeigen innerhalb kürzester Zeit hervorragende Resultate und Erscheinungsfreiheit. Selten bedürfen die Hämangiome aus ästhetischen Gründen einer Zweittherapie nach etwa 4 Wochen. Die therapiebedingte Depigmentierung der behandelten Stelle bei dunkelhäutigen Patienten repigmentiert sich wieder peu à peu.

Wenn die Kinder nach dem ersten Lebensjahr mit einem ausgewachsenen Hämangiom vorgestellt werden, erklären wir den Eltern, dass der richtige Zeitpunkt für eine effektive Therapie verpasst wurde, zeigen ihnen die bereits vorhandenen Zeichen der spontanen Regression und bitten sie, jetzt doch abzuwarten.

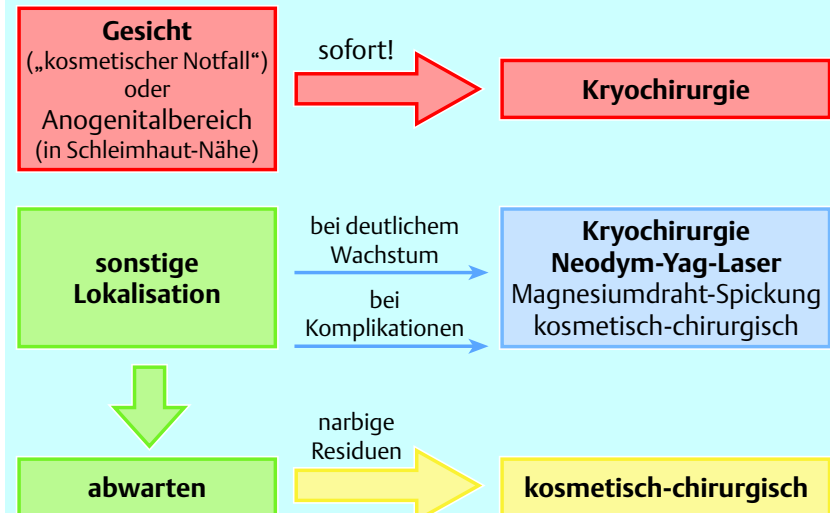

Abb. 3 Heilbronner Therapieschema der Säuglingshämangiome. 
Die vollständige spontane Regression der Säuglingshämangiome findet bei ca. 50\% dieser Hauterscheinungen statt und braucht Zeit. Bei ca. 30\% der Hämangiome bleiben Residuen übrig, die bei Vorhandensein von Therapiewünschen erst nach der Pubertät kosmetisch-chirurgisch angegangen werden sollten.

Es gibt leider Therapeuten, die planlos auch bei den eindeutig vorhandenen Regressionszeichen die zu spät vorgestellten Hämangiome behandeln. Außerdem werden immer wieder Modifikationen der Kryochirurgie vorgeschlagen, die nach meiner Kenntnis bisher nur eine Verlängerung der Modalitäten, Verzögerung der Heilung, Belastung der Familie durch häufige Einbestellung der Patienten zur Therapiewiederholung und dadurch die Erhöhung der Kosten (medizinische Leistungen, Fahrtkosten etc.) verursachen. Die Zerstückelung der Zeit (nur wenige Sekunden, dafür mehrere Male in wöchentlichen Abständen) bringt von der Schmerzempfindung her keinerlei Vorteile für die Patienten.

Die Vorteile der korrekt durchgeführten Kryochirurgie können wie folgt zusammengefasst werden:

- einfache Applikation bzw. Handhabung,

- ambulante Anwendbarkeit,

- kurze Behandlungsdauer,

- die Möglichkeit einer beliebigen Wiederholbarkeit,

- Durchführung ohne Lokalanaesthesie,

- Kostenersparnis,

- Schonung wichtiger anatomischer Strukturen,

- hervorragende kosmetische Resultate ohne Narbenbildung.

Als unerwünschte Nebenwirkungen sind Schmerzen während und kurz nach der Applikation der Kryochirurgie sowie Weichteilschwellungen (insbesondere im Gesicht) zu nennen.

\section{Abstract}

\section{The Current of the Early Treatment of Hemangiomas in Infancy}

In order to spare the patient cosmetic and psychological stress over a period of years, the hemangiomas in infancy are treated as soon as possible right at the onset when the hemangiomas are diagnosed. By omission of the early treatment the hemangiomas develop further complications. This kind of treatment is usual since over 15 years because of the very effective results instead of an attitude of wait-and-see.

\section{Literatur}

1 Bruckner AL, Frieden IJ. Hemangiomas of infancy. J Am Acad Dermatol 2003; 48: 477-493

2 Al Buainian H, Verhaeghe E, Dierckxsens L, Naeyaert JM. Early treatment of hemangiomas with lasers. Dermatology 2003; 206: 370-373

3 Cremer H, Djawari Dj. Frühtherapie der kutanen Hämangiome mit der Kontaktkryochirurgie. Pädiat Praxis 1994; 47: 633-650

4 Djawari Dj, Cremer $\mathrm{H}$. Kontaktkryochirurgische Frühbehandlung des Säuglingshämangioms. Akt Dermatol 1993; 19: 317-321

5 Djawari Dj, Barsom 0. Kryochirurgie, Renaissance einer effektiven Therapie. Akt Dermatol 1993; 19: 322 - 326

6 Djawari Dj. Zur Frühtherapie der Säuglingshämangiome. Z Hautkr 2002; 77: $14-18$

7 Haggstrom AN, Frieden IJ. Hemangiomas: Past, Present and Future. J Am Acad Dermatol 2004; 51: 50 - 52

8 Welsh 0, Olazarán Z, Gómez M, Salas J, Berman B. Treatment of infantile hemangiomas with short-term application of imiquimod $5 \%$ cream. J Am Acad Dermatol 2004; 51: 639-642
Buchbesprechung

Die Chaos-Zellen, Biologie der Krebserkrankung M. Reitz

Stuttgart: Hirzel, 2006. 220 S., 24 Abb. Kart. 19,80€. ISBN 3-7776-1433-5

Ein sehr nötiges und gut verständliches, handgerechtes und äußerst instruktives Buch liegt nun vor über Krebs aus der Feder eines promovierten Biologen und begnadeten Wissenschaftsjournalisten. Erfreulich und bestens geeignet für Laien, Patienten, deren Angehörige und für die Selbsthilfegruppen sowie zum Unterricht in den Schulen. Krebs gibt es bei Mensch und Tieren, schon nachgewiesen bei den Dinosauriern, den Vormenschen und schon bei unseren frühen Kulturvölkern. Jeder Krebs ist anders und tritt unterschiedlich häufig auf, die einen bei Kindern, die andern häufiger bei Erwachsenen. Und im Alter treten Krebserkrankungen immer häufiger auf. Sehr alten Menschen bleibt, seit wir nicht mehr an Infektionen sterben und die Herzkrankheiten behandelt werden können, dann kaum mehr eine Alternative zu entweder Krebs oder Demenz.

Krebs entsteht aus den Zellen und es gibt an die 200 verschiedenen Arten von jeder Zellsorte einen eigenen Krebs, dessen Zellen den ursprünglichen Zellen noch gleichen und doch enorme Abweichungen zeigen. Diese äußern sich in Form und Funktion, wodurch sie erkennbar sind, noch dem Ursprungsgewebe zugeordnet werden können und dennoch bizarre Egoisten darstellen. Sie wachsen rascher, in Phasen, aggressiv oder ruhend, jedoch ohne Rücksicht auf die Umgebung und sie zerstören was ihnen in den Weg kommt. Sie haben eigene Entwicklungen, ja sogar eigene Stammzellen, und verändern sich durch Anpassung und Auslese unentwegt. Dieses wird in glänzender Weise uns nahe gebracht und erklärt.

Erbliche Disposition, Exposition von „Onkogenen“, von Viren, und das Alter spielen zusammen, was am Beispiel von Lungenkrebs und Rauchen sowie Sonnenexposition und Hautkrebs erklärt wird. Die vielfältige Immunabwehr des Körpers wird uns gezeigt und das unentwegte Wechselspiel mit dem Gestaltwandel der Krebszellen, noch ergänzt durch die Effekte von Krebstherapien, erscheint in faszinierender Art vor dem Leser.

Ein wertvolles Buch fürwahr, dessen Zugang durch ein intelligentes Inhaltsverzeichnis sowie ein ausführliches und klares Glossar erschlossen wird. Zudem hilft ein gutes Sachregister.

Dieses Buch ist ein trefflicher Leitfaden für Patienten und alle Interessierte am Thema Krebs. Es fördert Verstehen, baut Fehldenken ab und begegnet den durch mangelnde Information bedingten Ängsten. Möge es vielen Gesunden dienen und den Kranken helfen!

E. G. Jung, Heidelberg 